\title{
Un estudio comparativo de las estrategias discursivas inglés-español utilizadas en la impartición de contenido en un contexto AICLE
}

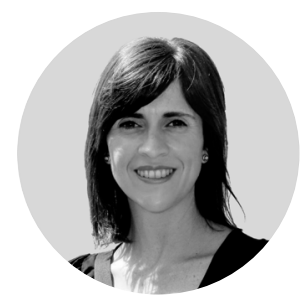

SANDRA PÉREZ COSTA

ESCUELA OFICIAL DE IDIOMAS CORDUBA sandra.perezcosta@gmail.com

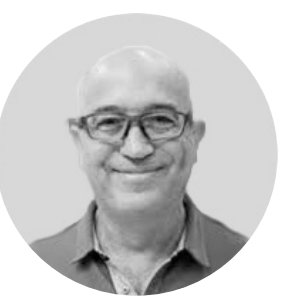

VÍCTOR PAVÓN VÁZQUEZ

UNIVERSIDAD DE CÓRDOBA

victor.pavon@uco.es
$\mathrm{L}$

a enseñanza de contenidos académicos a través de una lengua extranjera implica que el acceso al conocimiento mediante este medio produce un desarrollo de la lengua utilizada para la docencia y a la par contribuye a consolidar el conocimiento. No obstante, hay que tener en cuenta que cuando nos referimos a la lengua no solo lo hacemos con respecto a su dimensión conversacional, sino que también se incluye al lenguaje que se encuentra relacionado con el material académico. Así pues, el análisis de cómo se construye el significado en las áreas de conocimiento, se revela como un elemento esencial a la hora de describir los procesos de índole cognitiva que se producen en el alumnado durante la transmisión y manipulación del conocimiento académico. $\mathrm{Y}$, por ende, el estudio de las técnicas que se utilizan en el aula para manipular el conocimiento, se constituye también como un ámbito de investigación de indudable interés para entender estos procesos. En este estudio se han analizado las pautas de comunicación que se utilizan en dos lenguas, español e inglés, durante la impartición de una misma materia en la última etapa de enseñanza primaria con el fin de identificar qué tipo de técnicas son más comunes y cuáles son las diferencias que podemos encontrar en su uso en ambas lenguas.

PALABRAS CLAVE:

AICLE; lenguaje académico; construcción del significado; estrategias discursivas.

\begin{abstract}
Teaching academic content through a foreign language implies that this proposal fosters the development of the foreign language and contributes to the consolidation of academic content. However, it must be noted that the language comprises not only the conversational use but also the academic language that belongs to the content areas. Therefore, the study of how the meaning is constructed in these areas becomes a central element of analysis for the description of the cognitive processes at work during the transmission and manipulation of academic content. Moreover, the study of the techniques used in the classroom also becomes an interesting area in order to comprehend these processes. In this study we have analysed the communicative patterns used in English and Spanish during the teaching of similar content matter with the purpose of identifying the most frequent techniques used in both languages and finding out the most significant differences, if any.
\end{abstract}

KEYWORDS:

CLIL; academic language; construction of meaning; discourse strategies. 


\section{Introducción}

E l clima europeo de fomento de la promoción de las lenguas brinda, en la mayoría de los sistemas educativos, renovado protagonismo a la competencia comunicativa en todas sus facetas. Se promociona la competencia en segundas lenguas con la intención de trascender la función tradicional del aprendizaje de una lengua como la mera transmisión de significados. A nivel de producción, los esfuerzos se vuelcan en la promoción de medidas que atañen a aspectos tales como la calidad del 'input' del profesorado y su efecto sobre el alumnado; mientras que en el ámbito de la interacción y mediación el foco se sitúa en ámbitos tan variados como la conciencia multicultural y multilingüe o la creación conjunta del significado (Eurydice, 2001; Eurydice, 2003).

En este contexto, la enseñanza integrada de lengua y contenidos puede asemejarse a una situación más naturalmente comunicativa, en lo tocante a las demandas cognitivas que se plantean al alumnado. Existe acuerdo general en torno a la integración de los aspectos cultural, cognitivo y del contenido (Coyle, 2000), y se afirma que dicha integración repercute de forma positiva en la competencia del alumnado para comunicarse y desarrollar el pensamiento disciplinar cuando se usa una lengua nueva como vehículo de comunicación; al tiempo que la integración de estos tres elementos traerá aparejado el desarrollo de nuevas habilidades cognitivas relacionadas con el uso de la lengua. En este sentido, dentro de un contexto de bilingüismo educativo, particularmente en contextos de Aprendizaje Integrado de Lengua y Contenidos (AICLE), Pavón y Ellison (2013:72) abogan por un replanteamiento en lo que respecta a las pautas comunicativas del profesorado pues "no sería efectivo enseñar el mismo contenido /.../ usando otro idioma, sino contribuir a la compresión del contenido del alumnado a través del uso y manipulación de ese lenguaje". De ahí la importancia de que los docentes implicados deban estar dispuestos a reflexionar a priori no sólo sobre el lenguaje propio de la disciplina que imparten, sino también sobre el lenguaje funcional que será necesario poner en práctica y su impacto previsible, al tiempo que analizan los efectos que el empleo de esa técnica tiene sobre su propio alumnado.

Por estas y otras razones, resulta de especial interés analizar la interacción que se produce entre alumnado y profesorado, ya que es en la interacción donde presumiblemente se desarrolla el conocimiento; precisamente como comentan Ruíz de Zarobe y Jiménez (2009:198), en el "proceso dialógico entre expertos y aprendices, y entre iguales". No obstante, nuestro estudio vira ineludiblemente hacia el terreno del habla del profesorado y su potencial como herramienta comunicativa que, entendemos, se erige como inevitable ejemplo para el alumnado; y nos interesa aproximarnos tanto a la interacción que tiene lugar en contextos no bilingües, como aquella que se produce a través de una lengua vehicular que no es la materna del alumnado; no en vano, uno de los objetivos del Plan de Acción 2004-2006 (Eurydice, 2003) planteaba el desafío de estimulación de los docentes de las diferentes áreas de conocimiento curricular a enseñar alguna de sus asignaturas en, al menos, una lengua extranjera

La aproximación que haremos al discurso del profesorado será por medio del análisis de aquellas técnicas. En los contextos AICLE, la utilización de estrategias de guía adquiere una importancia capital dado que son las herramientas básicas para que se produzca la adquisición del conocimiento (Marsh, 2013; Méndez y Pavón, 2012). El análisis del lenguaje utilizado y su objetivo pedagógico será, por tanto, fundamental para poder establecer protocolos para que se produzca una utilización más efectiva. En este estudio se persigue establecer una comparación entre las formas de conversar que se exhiben en español y en inglés a la hora de trasmitir conocimientos de un mismo tipo y nivel, con el objeto de analizar las características de las técnicas utilizadas y de establecer los principios generales de su uso en una y otra lengua, puesto que las dos trabajan en conjunción desde la implementación de AICLE en los centros educativos, sobre todo a nivel cognitivo en la mente de los participantes en estos contextos.

\section{El empleo del lenguaje académico}

a clase es el contexto social donde, gracias a la interacción, las asignaturas escolares adquieren - significado (Dalton-Puffer, 2011) y en el aula AICLE esto se hace posible gracias a la atención prestada al lenguaje académico (Chamot y O’Malley, 1994; Mohan, Leung, y Davison, 2001; Zwiers, 2007) y gracias a la promoción de los distintos discursos derivados de las áreas de conocimiento (Dalton-Puffer, 2007a, 2007b; Lose, 2007; Smit, 2010). En el ámbito docente el lenguaje académico se manifiesta como una herramienta indispensable para favorecer la aproximación del alumnado al contenido de cada área curricular (Meyer et al., 2015).

Sin embargo, estos mismos estudios arrojan luz sobre las dificultades halladas por el alumnado para la adopción de este lenguaje. En primer lugar, porque el lenguaje académico ligado a cada área curricular no se encuentra claramente articulado en el diseño de los programas educativos. Al hallarse a similar distancia del lenguaje y del contenido, el lenguaje académico no suele ser enseñado de forma explícita (Coyle, 2015). Por otro lado, aún disponiendo de proyectos pioneros que tratan de categorizar el lenguaje académico según las disciplinas (Beacco, 2010; Polias, 2016; Martin y Rose, 2003, 2012; Grupo de Graz, 2014), al igual que ocurre en otros campos, no podemos esperar una utilización automática de un recurso determinado sin la correcta aproximación o guía, dificultad esta que ha de ser abordada desde el punto de vista de las demandas cognitivas que dicho reto plantea al alumnado.

La otra gran cuestión a la que nos lleva poner el foco en el contenido es cómo dicho contenido es generado durante la interacción alumnado-profesorado; si se trata de una transmisión, se trata de una creación conjunta, o bien consiste en algo diferente. Coyle (2000) plantea la dicotomía creación 
del significado (meaning-making) frente a transferencia de conocimiento (knowledge transfer) a modo de interrogante. La revisión de la bibliografía sobre este tema apunta al protagonismo indiscutible del alumnado en el proceso de generación de nuevos significados que, idealmente, ha de producirse en el aula. Si bien, a pesar a pesar de los esfuerzos encaminados hacia un enfoque más centrado en el alumnado, sabemos que el diseño de un enfoque integrado confiere gran protagonismo al docente, (Dalton-Puffer, 2008, 2011; Wilkinson, 2015), lo cual podría ser explicado por la necesidad del profesorado de tener mayor control sobre la nueva situación comunicativa a la que estaba haciendo frente o a una competencia más limitada en este ámbito.

Lo que parece claro es que el punto donde docente y alumnado se encuentran es clave para hallar la respuesta a muchas de nuestras preguntas. Más allá de la mayor o menor presencia del profesorado y la espontaneidad en sus intervenciones, lo realmente conveniente es maximizar los instantes de interacción a todos los ámbitos y del uso del lenguaje por parte del alumnado, pues en contextos socioculturales monolingües, el aula será en muchos casos el único escenario en donde el alumnado esté expuesto a este tipo de discurso (Dalton-Puffer y Smit, 2013) y las oportunidades de aprendizaje se verán maximizadas cuanto mejor sea la calidad de la interacción en clase (Walsh, 2012).

Otro concepto teórico que nos permite entender la interrelación entre el habla que el alumnado ya posee y la variedad que debería producir para un uso competente del lenguaje académico y, en fin, la creación mental del significado, fue descrito por Cummins $(1979,2000)$ a través de la dicotomía entre el lenguaje que se exhibe por medio de Destrezas de Comunicación Interpersonal Básica (BICS) y la Habilidad Cognitiva del Lenguaje Académico (CALP). Estas categorías nos permiten trazar la diferencia entre dos tipos de lenguaje, y nos capacitan para describir el lenguaje que el alumnado exhibe en el contexto comunicativo. Sin embargo, esta distinción no nos permite afirmar per se que se generen de forma lineal y uno sea base necesaria para el otro (Dalton-Puffer, 2013), aún cuando, según la descripción de Cummins, ambos son susceptibles de desarrollarse en paralelo siempre que se produzcan las condiciones necesarias en el contexto social indicado (Cummins, 2000:74; Meyer et al., 2015). La adquisición de esta capacidad cognitiva de reconocimiento y uso del lenguaje académico es pues uno de los objetivos del aula AICLE.

La adquisición de la competencia CALP no debe jugar a favor o en detrimento de la capacidad comunicativa en los diferentes idiomas de cada individuo (Lorenzo y Trujillo, 2017) sino que debe integrarse en su competencia multilingüe (Pavón, 2018). De modo que se plantea un nuevo objetivo, a saber, el fomento en el alumnado de la competencia para la integración del lenguaje académico también denominado Second Language Instruction Competence o SLIC (MacSwan y Rolstad, 2003). Esta competencia es también descrita más que por su faceta activa, por su relevancia para el pensamiento más profundo. Así, Meyer y Coyle
((2017:200) la definen como un proceso por medio del cual un individuo llega a ser capaz de transportar aquello que ha aprendido en una situación a otra nueva y aplicarlo. Para que esta aproximación al lenguaje académico sea factible, el papel del profesorado y su discurso, como guía al alumnado a través de la materia de conocimiento, son de especial relevancia. Es por ello que el docente en este ámbito de integración de contenidos y lengua debe ser consciente y selectivo en lo que respecta al lenguaje que utiliza para facilitar dicho acercamiento.

\section{Técnicas de comunicación utilizadas en contextos AICLE}

$\mathrm{E}$ l discurso en el aula es considerado, a grandes rasgos, un género discursivo propio, (Vygotski, 1962; Hammond, 1987; Mortimer y Scott, 2003; Drew y Heritage, 1992; Martin y Rose, 2003) y que está siendo producto de investigación constante (Christie, 2002; Cummins, 2007; Edwards y Westgate, 1994; Mercer, 1995, 1999; Walsh, 2006). Por ello y por las razones apuntadas anteriormente en lo que concierne al valor del lenguaje académico para la construcción del significado, resulta conveniente que se analicen las formas de conversar que tienen lugar en contextos de enseñanza integrada de lengua y contenidos.

Mercer (1995) ha resumido tres propósitos comunicativos en los cuales se puede englobar el uso de determinadas técnicas:

\section{- Obtener conocimiento de los estudiantes} (elicit knowledge from learners)

\section{- Responder a lo que dicen los estudiantes (respond to what learners say)}

\section{- Describir aspectos importantes de la experiencia compartida \\ (describe significant aspects of shared experiences)}

Cada uno de estos propósitos comunicativos fue identificado por medio del uso de una serie de pautas de comunicación que, según este autor, no responden al estilo personal del docente, sino que son producto de tradiciones culturales y de los escenarios en los cuales tienen lugar. Nos referiremos a estas formas de utilización del lenguaje como técnicas, de acuerdo con Mercer, quien también utiliza de forma menos precisa términos como "estrategias de guía", "pautas de comunicación" o "formas de conversar".

La aplicación comparativa del modelo analítico de Mercer en contextos similares en donde sólo se enseña utilizando la lengua madre podrá informarnos acerca de la tipología comunicativa de ambos contextos situacionales, lo que nos permitirá realizar no sólo la descripción, sino el análisis contrastivo de los discursos del profesorado en una y otra lengua. 
Mercer (1995) también determina cómo el lenguaje es utilizado en colectividad para construir significado a través de las actividades del aula, y destaca la relevancia del diálogo para generar conocimiento. En su estudio y en investigaciones afines (Edwards y Westgate, 1994; Edwards y Mercer, 1988; Mercer 2004) se hace patente la trascendencia que la selección de elementos por parte de los hablantes posee a nivel funcional, a la par que se muestra la forma en la que los docentes presentan los acontecimientos y la organización de los mismos. Este fenómeno es descrito como una estrategia que, utilizada con la sistematicidad necesaria, puede convertirse en una técnica pedagógica. Su uso reflexivo e intencional consiste en una mezcla de planificación tanto a nivel metodológico como discursivo, ambos dirigidos a amplificar las oportunidades de aprendizaje del alumnado (Van Lier, 1996; Moore, Márquez y Gutiérrez, 2014).

\section{Diseño de la investigación}

\subsection{Objetivos}

E estudio pretende identificar las técnicas de conversar que el profesorado exhibe en determinadas ocasiones, lo cual permitirá describir la tipología comunicativa usada en la asignatura de Conocimiento del Medio impartida en español en el aula de primaria y establecer una comparativa con la asignatura impartida en inglés en un contexto AICLE. El estudio analizará la mayor o menor frecuencia de uso de dichas técnicas y el propósito comunicativo a que responden, así como casos en los que su utilidad pueda ponerse en duda. Para ello, partimos de las siguientes preguntas de investigación:

\section{P.I.1. ¿Qué estrategias de guía están presentes en el habla del profesorado de la última etapa de educación primaria en contextos donde se utiliza el español (L1) y en contextos comparables donde la asignatura se imparte en inglés (L2)?}

\section{P.I.2. ¿Existen similitudes en el tipo y frecuencia de uso de las técnicas discursivas utilizadas en ambas lenguas?}

\subsection{Contexto del estudio}

E lestudio ha sido llevado a cabo a través de la observación de una única disciplina, Conocimiento del Medio, en la última etapa de Educación Primaria durante el tercer trimestre de los cursos académicos 2015/16 y 2016/17 en 4 centros escolares de la capital de Córdoba. En la selección de la población se optó por recurrir a una división natural. En estos últimos estadios de la enseñanza primaria, conducentes a secundaria, sería donde el alumnado comienza a manifestar un manejo de habilidades menos básicas y más específicas de las diferentes disciplinas (Shanahan y Shanahan, 2008:44) no sólo en la lengua meta, sino también en la lengua materna.

Respecto a la selección de los centros, se buscó aquellos en los que la implementación del programa bilingüe tuviera similar grado de desarrollo (López Morillas, 2011; Ortega, 2011), a fin de encontrar contextos comparables de docencia en L1 (la lengua materna del alumnado, el español) y L2 (la lengua adicional utilizada como vehículo de instrucción en algunas asignaturas, el inglés). Además de ello, se procuró atender a un criterio inclusivo, que pudiera minimizar el sesgo de la propia selección. En particular, para el proceso de observación, se obtuvieron muestras tanto de centros de la red pública, como de la red concertada y que estuvieran distribuidos por diferentes zonas de la misma ciudad a fin de poder trabajar con diferentes perfiles socio-educativos y alumnado perteneciente a familias de variado estatus socioeconómico.

Los docentes cuyas clases fueron observadas fueron 6 , todos ellos en centros que pertenecen a la red de centros bilingües de la Comunidad Autónoma andaluza, lo cual implicaba que cuentan con una línea denominada bilingüe, donde determinadas asignaturas, como es el caso de Conocimiento del Medio, se imparten en inglés. Su pertenencia a la Red de Centros Bilingües también suponía una homogeneidad en el enfoque e implementación de estas líneas bilingües, dado que todos ellos comenzaron su andadura al amparo del Plan de Fomento del Plurilingüismo (2005) y cada centro contaba con una persona encargada de la Coordinación de las unidades bilingües dentro del Centro. Una entrevista previa con los docentes nos informaba de que su dedicación a la docencia superaba los 10 años. Todos los que impartían sus clases en L2 eran especialistas en lengua inglesa y contaban con la asistencia de un auxiliar de conversación en clase, aunque no necesariamente en todas las sesiones, siendo diferentes las funciones que dicho auxiliar realizaba en cada centro y clase. Los docentes que usaban la L2 habían impartido o bien se encontraban impartiendo clases en L1 en otros niveles de primaria.

\subsection{Instrumentos de análisis y recolección de datos}

as transcripciones que aquí presentamos son parte de un corpus de 6 horas aproximadas de grabación, tres de ellas (D1, D2 y D3) corresponden a docentes de Conocimiento del Medio en español (ESP-L1) y las otras tres (D4, D5 y D6) a los docentes de Social and Natural Science en inglés (ING-L2). Estas grabaciones se realizaron durante el desarrollo de una unidad temática de las citadas asignaturas en el tercer trimestre y en dos cursos de forma paralela, en $4^{\circ}$ y $6^{\circ}$ de Educación Primaria de la Red de Centros Bilingües públicos y concertados de la capital cordobesa.

El papel de la investigadora durante las grabaciones de las clases fue de observadora silenciosa. Su presencia en el aula se limitó a la observación y grabación de las clases 
para posteriormente, proceder a la transcripción de las grabaciones.

Para la transcripción de las sesiones seguimos la tradición de transcripción utilizada por Edwards y Mercer (1988) y Mercer (2004), con la intención de reflejar fielmente lo que se ha dicho, a fin de contar con el mayor número de elementos relevantes para el análisis y que las contribuciones de los hablantes no estén sujetas a interpretaciones. Este sistema se resume en los elementos reseñados en la Tabla 1.

Tabla 1. Simbología de la transcripción

\begin{tabular}{ll}
\hline$\ldots$ & la secuencia empieza o termina \\
\hline$/$ & pausa de menos de 2 segundos \\
\hline Negrita & pausa de más de 2 segundos \\
\hline[ & Habla simultánea o interrumpida \\
\hline \&) & $\begin{array}{l}\text { Habla continuada, separada en la } \\
\text { transcripción por la interrupción }\end{array}$ \\
\hline
\end{tabular}

Con el fin de analizar las transcripciones se ha recurrido a una taxonomía previamente validada (Mercer, 2004) a fin de analizar las pautas de comunicación que tienen lugar en el aula. A diferencia de sus primeras versiones (Mercer, 1995, 1999), en ésta se incluyen aspectos tales como la exhortación, la cual nos parece de relevancia.

De este modo en Respuesta a lo que dicen los estudiantes buscaremos ejemplos de:

\section{A Confirmaciones \\ B Repetición a modo de eco \\ C Repetición correctiva \\ D Elaboraciones}

\section{Para la Descripción de aspectos importantes de la experiencia} compartida:

\section{E Exhortaciones \\ F Recapitulaciones}

Los recursos lingüísticos que analizamos son formas de utilización del lenguaje las cuales, según el análisis de Mercer (2004), el profesorado pone en práctica de manera más o menos espontánea durante intercambios interactivos con su alumnado de forma generalizada en diferentes lugares. Como se podrá observar por los ejemplos, ninguna de estas técnicas tendría sentido si no fuera porque busca la generación de significados con el propio alumnado, y, en la mayoría de los casos, se apoya en los turnos de palabra del alumnado para determinar la continuidad o no de la estrategia. Lo relevante de esta selección de técnicas es su producción en un contexto interactivo y con un propósito determinado, que será reforzado o abandonado, en función del resultado obtenido en la interacción.

\section{Presentación y análisis de resultados}

La categorización de las formas de conversar de los docentes según la taxonomía de Mercer (2004) arrojó los siguientes resultados:

Respecto a la ocurrencia de las técnicas que anticipamos con anterioridad, introducimos una tabla resumen:

\begin{tabular}{lcc}
\hline & $\begin{array}{c}\text { L1 } \\
\text { (Spanish) } \\
\text { Discourse } \\
\text { strategy } \\
(\%)\end{array}$ & $\begin{array}{c}\text { L2 } \\
\text { (English) } \\
\text { Discourse } \\
\text { strategy } \\
(\%)\end{array}$ \\
\hline CONFIRMACIONES & 59,10 & 40,90 \\
\hline REPETICIÓN A MODO DE ECO & 49,70 & 50,30 \\
\hline REPETICIÓN CORRECTIVA & 0 & 90,90 \\
\hline ELABORACIÓN & 42,90 & 57,10 \\
\hline EXHORTACIONES & 59,20 & 40,80 \\
\hline $\begin{array}{l}\text { RECAPITULACIONES } \\
\text { CONSTRUCTIVAS }\end{array}$ & 90,10 & 90,90 \\
\hline
\end{tabular}

A continuación, presentamos ejemplos extraídos del corpus de transcripciones donde se identifican las técnicas que han sido utilizadas y el objetivo que perseguían. Siempre que es posible, cada categoría se ilustra con un ejemplo de aula ESP-L1 y uno de aula ING-L2. 


\section{A}

\section{Confirmaciones}

Este intercambio es típico de una clase y revela la singularidad de la interacción en dicho contexto, en la medida en la que, en la vida real, un hablante no esperaría este tipo de apreciación por parte de su interlocutor.

\section{Fragmento 1, D4}

1. Teacher: Poultry? Yes! Eh, that's a good word. Poultry. Who remembers what poultry means?

2. Student: Aves de corral.

3. Teacher: Yes, very good.

\section{Fragmento 2, D3}

1. Profesor: El foro, ¿que era el foro?

2. Alumno: Era lo del centro de la ciudad.

3. Profesor: ¿Y para que servía?

4. Alumnos: Servia pues para

5. Profesor: [Javier, ¿para que servía el foro?

6. Alumno: Para reunirse

7. Profesor: Muy bien, lugar de reunión

$B$

\section{Repetición a modo de eco}

La interacción entre alumnado y profesorado halla también una forma muy visible de extenderse, a través de la repetición, a modo de eco, por parte del profesorado, de palabras literales que el alumnado haya usado. El recurso de esta técnica por parte del profesorado en cierto modo valida la contribución del alumnado, convirtiéndose en otra forma de confirmar la información ofrecida. Como se puede observar se encuentra en el discurso del profesorado en ambas lenguas.

\section{Fragmento 3, D4}

1. Teacher: What does that mean? If housing was temporary, what does that mean? They had a house in the same place or time?

2. Students: No.

3. Teacher: No. They had to?

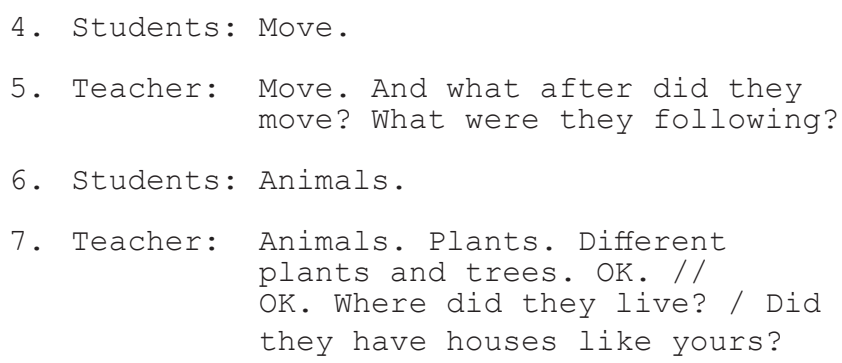

\section{Fragmento 4, D3}

1. Profesor: Bien, los colonizadores de la Península Ibérica. ¿Quién me puede hablar de los tres grandes grupos, Jorge?

2. Alumno: Primero fueron los fenicios

3. Profesor: Primero los fenicios

4. Alumno: Luego los griegos

5. Profesor: Griegos ¿y?

6. Alumno: Y luego los cartagineses

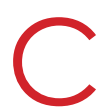

\section{Repetición correctiva}

La repetición puede llevar consigo otras funciones más concretas. En algunos casos, el docente se vale de la repetición con intención de corregir la producción del alumnado. En el ejemplo que incluimos a continuación observamos la coexistencia de dos tipos de propósitos correctivos; uno de ellos atañe a la corrección gramatical, y el otro a un deseo por corregir la pronunciación de un término.

\section{Fragmento 5, D4}

1. Teacher: ... Another metal, very good.

2. Student: They start Leyendo

3. Teacher: [Started

4. Student: Started using bronze wea Leyendo

5. Teacher: [Weapons

6. Student: Weapons and tools that were stronger than copper. Leyendo

7. Teacher: Copper ones. OK, so there's another evolution.

Esta estrategia discursiva no ha encontrado correspondiente en los ejemplos con ESP-L1. 
<smiles>C1CCCC1</smiles>

\section{Elaboración}

Este recurso se localiza en aquellas ocasiones en las que el docente decide extender la contribución del alumnado, de forma que aparezca más elaborada o acorde con el propósito comunicativo. Ofrece una variante cercana a la expresión utilizada por el alumnado, pero que acarrea algún matiz diferenciador, que puede responder a diversos propósitos específicos del instante. En el siguiente ejemplo de las sesiones en español, el grupo estaba comparando una serie de cuerpos según su volumen, peso y masa.

\section{Fragmento 6, D2}

1. Profesor: Madera de balsa. Es una madera que pesa muy poquito, es resistente, pesa poquísimo. Y cuando se le coloca un motorcito pequeñito, el avión puede volar porque pesa muy poco. Si pesase mucho no podrían. Bueno, ¿lo entendemos?

2. Alumnos: Si.

3. Profesor: y si tuviésemos un taco de madera de balsa equivalente al de folio, ¿que pesaría más?

4. Alumnos: El folio.

5. Profesor: El papel con grandísima diferencia

Si bien el profesor introduce un ejemplo concreto para ejemplificar la explicación, técnica de la que el alumnado hace uso, como se puede apreciar por la repetición del alumnado del término usado por el profesor ('el folio'), una vez que el docente percibe que el alumnado ha elaborado el discurso específico que se ejemplificaba, inmediatamente parafrasea la respuesta de modo que el término que introduce abandona el ámbito concreto del ejemplo para validar el conocimiento generado de modo general. En las lecciones en inglés se halla la presente técnica en el mismo sentido, si bien también puede responder a un uso similar a la repetición correctiva que vimos en el anterior apartado. Observando el siguiente ejemplo percibimos las dos.

\section{Fragmento 7, D4}

1. Students: Metal workers

2. Teacher: The metal workers. And what did they make?

3. Students: Tools. Respuestas simultáneas

4. Teacher: And weapons, for him?

5. Students: Everyone.

6. Teacher: For everyone

\section{Exhortaciones}

El docente hace explícito su interés por demostrar la continuidad del hilo informativo por medio de exhortaciones a que recuerden, piensen o usen su lógica; el recurso a esta técnica parece orientado a servir al alumnado como hilo conductor entre la lección anterior y la actual, de forma que las perciban conectadas y con un desarrollo coherente.

\section{Fragmento 8, D4}

1. Teacher: And, well they painted hands on the walls, very old walls. Very good. It's like

they printed, yes? What else do we know about Paleolithic? Or do you remember?

2. Student: The / tools were with stone, wood and bones. Leyendo

3. Teacher: OK. Stone / wood, and / what you said more?

4. Student: Bones.

5. Teacher: Bones. OK, very good. And, erm, what else? / Someone else.

6. Student: People learned to use fire Levanta la mano Leyendo

Observamos su existencia también en las lecciones en español.

\section{Fragmento 9, D2}

1. Profesor: El volumen. No podemos pensar en un papelito solo, en un papelito pequeño. Si no, en una cantidad de papel equivalente a ese mismo volumen que los otros cuerpos. Si tenemos ese volumen de plumas, ¿eso pesa mucho?

2. Alumnos: No

3. Profesor: Un volumen de plumas, por ejemplo, un edredón. ¿Todos 10 habéis cogido, verdad?

4. Alumnos: Si.

5. Profesor: Estos edredones que hay de plumas, ¿eso pesa mucho?

6. Alumnos: No.

7. Profesor: ¿Para el tamaño que tiene?

8. Alumnos: No.

9. Profesor: Apenas, ¿verdad? No pesa casi nada. Ahí está claro que no. 


\section{F}

\section{Recapitulaciones}

Las sesiones comienzan con una recapitulación constructiva en la que el docente va preguntando al alumnado por información relacionada con el impacto del ser humano sobre el hábitat natural o con los colonizadores de la península ibérica en época prerromana. Con el fin de reforzar algunas de las ideas que se comparten, se hacen referencias a información anecdótica común tanto para el profesor como por el alumnado. Así mismo, se explicitan referencias a sus experiencias comunes recientes. Parte del alumnado se encuentra más seguro con el uso de la lengua en esta etapa, atreviéndose a utilizar lenguaje de forma más autónoma y menos guiada por el profesorado, pues se trata de una revisión de conocimiento que se compartió en sesiones anteriores y, como se puede apreciar por la contribución que hace el profesorado en cada interacción, la intención de estos intercambios no es la de introducir nuevo conocimiento a la conversación, sino comprobar que el alumnado ya está en posesión de dicha información.

\section{Fragmento 10, D2}

1. Profesor: ... No. Las centrales hidroeléctricas, ¿eran muy contaminantes? ¿Eran de las que producían mucho impacto, o más bien limpias?

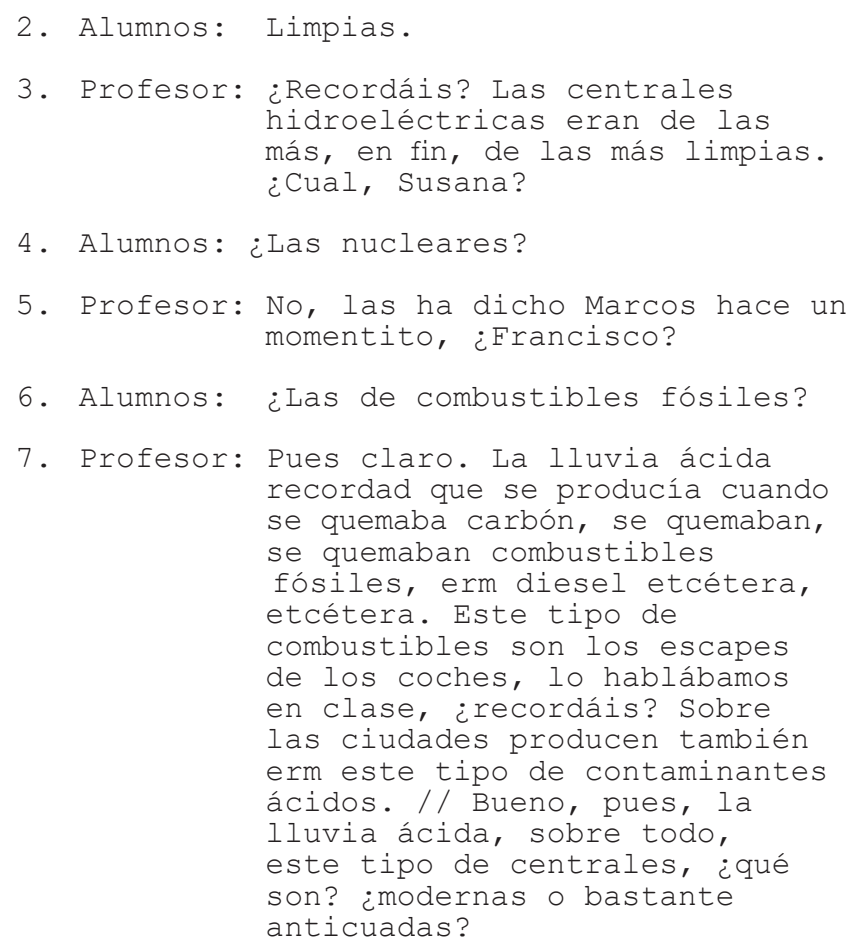

4. Alumnos: ¿Las nucleares?

5. Profesor: No, las ha dicho Marcos hace un momentito, ¿Francisco?

6. Alumnos: ¿Las de combustibles fósiles?

7. Profesor: Pues claro. La lluvia ácida recordad que se producía cuando se quemaba carbón, se quemaban, se quemaban combustibles fósiles, erm diesel etcétera, etcétera. Este tipo de combustibles son los escapes de los coches, lo hablábamos en clase, ¿recordáis? Sobre las ciudades producen también erm este tipo de contaminantes ácidos. // Bueno, pues, la lluvia ácida, sobre todo, este tipo de centrales, ¿qué son? ¿modernas o bastante anticuadas?

\section{Alumnos: Anticuadas}

\section{Fragmento 11, D3}

1. Teacher: Very good. So then, Paleolithic went into evolution and we went into another period which was? Gesticula con las manos

2. Students: // Mesolithic. Respuestas simultáneas

3. Teacher: And well, do we know much about Mesolithic period? It was a.. Gesticula con las manos

4. Students: [Transition. Respuestas simultáneas

\section{Discusión}

A la luz de los datos obtenidos procedemos a contestar las preguntas de investigación planteadas.

\section{P.I.1. ¿Qué estrategias discursivas están presentes en el habla del profesorado de la última etapa de educación primaria en contextos donde se utiliza el español (L1) $y$ en contextos comparables donde la asignatura se imparte en inglés (L2)?}

Se ha constatado el uso de las confirmaciones en respuesta a las intervenciones de los estudiantes tanto en las lecciones en español como en inglés, un hecho común en otros contextos investigados (Frohlich, Spada y Allen, 1985). En lo que concierne a la repetición de palabras inmediatamente después de haberlas emitido el alumnado, esta estrategia se encuentra presente en las dos lenguas y en ocasiones se ha detectado que perseguía un propósito opuesto al de validar lo dicho, ya que se trataba de ofrecer una corrección. Con respecto a la repetición correctiva, en la que el docente repite la palabra que utiliza el alumnado, no ofreciendo una expresión alternativa, es decir, no parafraseando, sino repitiendo, al tiempo que mejora un determinado aspecto de la producción, no se han encontrado ejemplos correspondientes en español. En cuanto a la elaboración, cuya diferencia con la estrategia anterior estriba en que no es simplemente una repetición o incluso eco, ya que requiere la introducción de algún elemento más que el alumnado ha olvidado introducir, su uso es poco común en ambas lenguas. Por lo que respecta a las exhortaciones, cuyo propósito primario encontrado es consolidar el conocimiento de lo aprendido en la lección anterior y estimular el pensamiento hacia el futuro o hacia lo hipotético y no acontecido, se ha observado un uso de esta estrategia en las dos lenguas. Por último, la estrategia de recapitulación, en la que la referencia a conocimiento previo ayuda a generar ideas colectivas, se encuentra presente en el discurso producido en las dos lenguas, de forma particular, además, en el momento final de la lección, durante la asignación del trabajo individual y el trabajo de casa. 
La segunda pregunta de investigación halla respuesta a través del análisis cuantitativo de los datos:

\section{P.I.2. ¿Existen similitudes en el tipo y frecuencia de uso de las estrategias discursivas utilizadas en ambas lenguas?}

El análisis de frecuencia de las técnicas exhibidas en el discurso del profesorado revela que, en cada lengua analizada, la mayoría de las pautas comunicativas utilizadas para interactuar con el alumnado con los propósitos que hemos observado se mueven en un rango similar de frecuencia a excepción de las Recapitulaciones Constructivas, la cuales cobran mayor protagonismo en las lecciones impartidas en inglés. Los datos obtenidos sugieren que el uso de las técnicas en las dos lenguas favorece un acercamiento al área desde el punto de vista de la dimensión social y cultural que ofrece el conocimiento del área como marco. En este punto es donde tiene relevancia la creación del conocimiento compartido (Mercer, 2004), donde el docente es el responsable de maximizar el uso de las pautas comunicativas con un propósito determinado: "CLIL teachers need provide learners with tailor-made assistance which will help them comprehend, produce and negotiate academic messages" (Escobar y Evnitskaya, 2013:125). La construcción que cada individuo lleve a cabo comienza con la interacción con el profesor para continuar con la interacción con los otros, llegando de este modo a la dimensión social y cultural. El paso de una a otra se lleva a cabo con técnicas visibles de razonamiento que los estudiantes utilizan para crear significado de forma conjunta (Madrid, 2011).

\section{Conclusiones}

E n este estudio se planteaba la necesidad de indagar en las pautas comunicativas del profesorado en lo que concierne a la transmisión de contenidos académicos de una misma asignatura en inglés y en español, con el fin de establecer una comparación en cuanto a su uso en ambas lenguas y así poder identificar su tipología y frecuencia. De acuerdo con los resultados obtenidos se puede concluir que, salvo en el caso de la corrección, técnica que no se ha detectado en español, y en el caso de la recapitulación, cuya frecuencia de uso ha sido menor, la mayoría de las técnicas tienen unas características similares en ambas lenguas. Se trata de una primera conclusión coincidente con otros estudios en contextos similares en los que las estrategias de comunicación no parecen diferir entre la lengua materna de los aumnos y la lengua extranjera utilizada como venículo de comunicación (Moore y Nikula, 2016), donde la principal diferencia estriba en el grado de competencia lingüística en esta lengua extranjera y no en en la utilización de las técnicas en sí (Lo, 2015; Pavón y Ramos, 2019).

Los esfuerzos dedicados a promover la integración del lenguaje académico en el discurso del aula de forma sistemática implican una forma de planificación más consistente (MacSwan y Rolstad, 2003) y cuyos efectos serán de utilidad en una diversidad de contextos. Pero coincidimos con quienes apuntan que aún es preciso mayor trabajo en este ámbito y que las clases AICLE aún muestran un claro déficit en el uso de recursos comunicativos de este tipo (Dalton-Puffer, 2007, 2011; Nikula et al., 2016; Meyer y Coyle, 2017). Por ello resulta relevante que se indague en el uso del lenguaje académico y en el de las técnicas discursivas que se utilizan para manipularlo en estos contextos, en los que la competencia lingüística del alumnado supone una de las variables más influyentes para el éxito o fracaso en la consecución de los objetivos de aprendizaje. Este estudio en particular ha pretendido indagar en estos aspectos y hacerlo además desde una perspectiva comparativa, no exenta de limitaciones derivadas del tamaño de la muestra analizada; por lo que sería conveniente seguir en esta línea de investigación y que se pudieran analizar más horas, más asignaturas, más niveles y más contextos para seguir aportando datos que ayuden a entender la tipología de uso de las diferentes pautas de comunicación en el aula.

\section{Bibliografía}

Beacco, J.C. (2010). Items for a description of linguistic competence in the language of schooling necessary for teaching/learning history (end of obligatory education). An approach with reference points. Estrasburgo: División de Políticas Lingüísticas. Consejo de Europa.

Chamot, A. y O'Malley, J.M. (1994). The CALLA handbook: Implementing the Cognitive Academic Language Learning Approach. Reading, MA: Addison-Wesley Publishing.

Christie, F. (2002). Classroom Discourse Analysis. A functional perspective. Londres: Continuum.

Coyle, D. (2000). "Meeting the challenge: developing the 3 Cs curriculum". En S. Green (ed). New perspectives on teaching and learning modern languages (págs. 158182). Clevedon: Multilingual matters.

Coyle, D. (2015). "Strengthening integrated learning: Towards a new era for pluriliteracies and intercultural learning". Latin American Journal of Content and Language Integrated Learning, 8(2), 84-103.

Cummins, J. (1979). "Linguistic interdependence and the educational development of bilingual children". Review of Educational Research, 49, 222-251.

Cummins, J. (2000). Language, power, and pedagogy: Bilingual children in the crossfire. Clevedon: Multilingual Matters.

Cummins, J. (2007). "Rethinking monolingual instructional strategies in multilingual classrooms". Canadian Journal of Applied Linguistics, 10(2), 221-240.

Dafouz, E. (2011). "English as the medium of instruction in Spanish contexts: A look at teacher discourses". En Y. Ruiz de Zarobe, J.M. Sierra y F. Gallardo del Puerto 
(eds.) Content and Foreign Language Integrated Learning. Contributions to Multilingualism in European Contexts (págs. 189 - 205). Berna: Peter Lang.

Dalton-Puffer, C. (2007a). Discourse in content and language integrated learning (CLIL) classrooms. Amsterdam, Países Bajos: John Benjamins.

Dalton-Puffer, C. (2007b). "Academic language functions in a CLIL environment”. En D. Marsh y D. Wolff (eds.). Diverse contexts - converging goals (págs. 201-210). Frankfurt: Peter Lang.

Dalton-Puffer, C. (2008). "Outcomes and processes in content and language integrated learning (CLIL): Current research from Europe.” En W. Delanoy y L. Volkmann (eds.), Future perspectives for English language teaching (págs. 139-157). Heidelberg: Carl Winter.

Dalton-Puffer, C. (2011). “Content-and-language integrated learning. From practice to principles? "Annual Review of Applied Linguistics, 31,182-204.

Dalton-Puffer, C. y Smit, U. (2013). "Content and Language Integrated Learning: A research agenda". Language Teaching, 46(04), 545-559.

Drew, P. and Heritage, J. (eds) (1992). Talk at Work. Cambridge: Cambridge University Press.

Edwards, D. y Mercer, N. (1998). El conocimiento compartido. El desarrollo de la comprensión en el aula. Barcelona: Paidós.

Edwards, D. y Westgate, D. (1994). Investigating Classroom Talk. Londres: The Falmer Press.

Escobar Urmeneta, C. y Evnitskaya, N. (2013). “Affording students opportunities for the integrated learning of content and language. A contrastive study on classroom interactional strategies deployed by two CLIL teachers. En J. Arnau (ed.), Reviving Catalan at School: Challenges and Instructional Approaches (págs. 158-182). Bristol: Multilingual Matters \& Institut d'Estudis Catalans.

Eurydice. Comisión Europea (2001). La Enseñanza de las Lenguas Extranjeras en el Contexto Escolar Europeo. Dirección General de Educación y Cultura.

Eurydice. Comisión Europea (2003). Promover el aprendizaje de idiomas y la diversidad lingüistica: Plan de Acción 2004-2006. (Visitado en Julio 2018: https:// publications.europa.eu/en/publication-detail/-/publication/ b3225824-b016-42fa-83f6-43d9fd2ac96d/language-en)

The Graz Group (2014). Literacies through content and language integrated learning: Effective learning across subjects and languages. Council of Europe, European Centre for Modern Languages.

Lo, Y-Y. (2015). "How much L1 is too much? Teachers' language use in response to students' abilities and classroom interaction in Content and Language Integrated Learning". International Journal of Bilingual Education and Bilingualism, 18(3), 270-288.
Lorenzo, F. y Trujillo, F. (2017). "Languages of schooling in European policymaking: present state and future outcomes". European Journal of Applied Linguistics, 5(2), pp. 177-197.

Lose, J. (2007). "The language of scientific discourse: Ergebnisse einer empirisch-deskriptiven Interaktionsanalyse zur Verwendung fachbezogener Diskursfunktionen im bilingualen Biologieunterricht”. En D. Caspari, W.Hallet, A. Wegner y W. Zydatiß (eds.), Bilingualer Unterricht macht Schule. Beiträge aus der Praxisforschung (págs. 97-107). Frankfurt am Main: Peter Lang.

MacSwan, J. y Rolstad, K. (2003). "Linguistic diversity, schooling, and social class: Rethinking our conception of language proficiency in language minority education. En Paulston, C.B. y G.R. Tucker (eds.), Sociolinguistics: The essential readings (págs. 329-340). Oxford: Blackwell.

Madrid, D. (2011). "Monolingual and bilingual students' competence in Social Sciences". En D. Madrid y S. Hughes (eds.) Studies in Bilingual Education (págs. 195222). Suiza: Peter Lang AG.

Martin, J.R. y Rose D. (2003). Working with Discourse: Meaning Beyond the Clause. Londres: Continuum.

Martin, J.R. Y Rose, D. (2012). Learning to Write, Reading to Learn: Genre, Knowledge and Pedagogy of the Sydney School. Sheffield: Equinox Publishing.

Mercer, N. (1995). The Guided Construction of Knowledge. Clevedon: Multilingual Matters.

Mercer, N. (1999). "Classroom language”. En B. Spolsky (ed.), Concise Encyclopedia of Educational Linguistics (págs. 315-319). Amsterdam: Elsevier.

Mercer, N. (2004). "Sociocultural discourse analyses: analysing classroom talk as a social way of thinking". Journal of Applied Linguistics, 1(2),137-168.

Méndez, M.C., y Pavón, V. (2012). "Investigating the coexistence of the mother tongue and the foreign language through teacher collaboration in CLIL contexts: perceptions and practice of the teachers involved in the plurilingual programme in Andalusia”. International Journal of Bilingual Education and Bilingualism, 15, 573-592.

Meyer, O. y Coyle, D. (2017). "Pluriliteracies Teaching for Learning: conceptualizing progression for deeper learning in literacies development". European Journal of Applied Linguistics, 5(2), 199-222.

Meyer, O., Coyle, D., Halbach, A., Schuck, K. y Ting, T. (2015). "A pluriliteracies approach to content and language integrated learning - mapping learner progressions in knowledge construction and meaningmaking". Language, Culture and Curriculum, 28(1), 4157.

Mohan, B. A. (1986). Language and Content. Reading, MA: The Addison-Wesley Publishing Company, Inc. 
Mohan, B., Leung, C. y Davison, C. (eds.). (2001). English as a Second Language in the Mainstream: Teaching, Learning and Identity. Harlow: Longman.

Moore, P., Márquez, M. y Gutiérrez, V. (2014). “La optimización del discurso del aula en el aprendizaje integrado de contenidos y lengua". Magazin/Extra, 1, 44-49.

Moore, P. y Nikula, T. (2016). "Translanguaging in CLIL". En T. Nikula, E. Dafouz, P. Moore and U. Smit (Eds.), Conceptualising Integration in CLIL and Multilingual Education (págs. 211-234). Clevedon: Multilingual Matters.

Mortimer, E. y Scott, P. (2003). Meaning Making in Secondary Science Classrooms. McGraw-Hill International.

Nikula, T., Dafouz, E., Moore, P., y Smit, U. (eds.) (2016). Conceptualising Integration in CLIL and Multilingual Education. Bristol: Multilingual Matters.

Plan de Fomento del Plurilingüismo: hacia un nuevo modelo metodológico. (2005) Consejería de Educación. Junta de Andalucía.

Pavón, V. (2018). "Innovations and challenges in CLIL research: Exploring the development of subject-specific literacies", Theory Into Practice, 57(3), 204-211.

Pavón, V. y Ellison, M. (2013). "Examining teacher roles and competences in Content and Language Integrated Learning (CLIL)". Linguarum Arena, 4, 65-78.

Pavón, V. y Ramos, M.C. (2019). "Describing the use of the L1 in CLIL: analysing students' L1 communication strategies in classroom interaction". International Journal of Bilingual Education and Bilingualism, 22(1), $35-48$.

Polias, J. (2016). Apprenticing students into science: doing, talking and writing scientifically. Melbourne: Lexis Education

Ruíz de Zarobe, Y. y Jiménez, R. (eds.), (2009) Content and Language Integrated Learning: Evidence from Research in Europe. Bristol: Channel View Publications.

Smit, U. (2010). "CLIL in an English as a lingua franca classroom: On explaining terms interactively". En C. Dalton-Puffer, T. Nikula y U. Smit (eds.), Language use and language learning in CLIL classrooms (págs. 259277). Amsterdam: Benjamins.

Van Lier, L. (1996). Interaction in the Language Curriculum: Awareness, Autonomy and Authenticity. Londres. Longman.

Walsh, S. (2006). Investigating Classroom Discourse (Domains of Discourse). Londres: Routledge.

Wilkinson, D. (2015). "English-Medium content courses: student approaches and strategies to increase comprehension levels". International Journal of Learning, Teaching and Educational Research, 11(3), 1-16.
Zwiers, J. (2007). "Teacher practices and perspectives for developing academic language." International Journal of Applied Linguistics, 17, 93-116. DOI: https://doi. org/10.1111/j.1473-4192.2007.00135.x 